\title{
Correction \\ Ergodic and Adaptive Control of Nearest-Neighbor Motions
}

Vivek S. Borkar and Mrinal K. Ghosh

In [BG] the proof given for Theorem 4.1 is incorrect. The same proof is reproduced in [B]. The following correct argument uses the notation of [BG].

If $K>0$ is a bound on $\mid\left(k(\cdot, \cdot) \mid,(3.2)\right.$ implies that $\left|V_{\theta}(i)\right| \leq 2 K E_{\theta}\left[\tau(0) \mid X_{0}=i\right]$. From Corollary 3.13, it follows that $\sup _{\theta}\left|V_{\theta}(i)\right|<\infty$ for each $i$. The rest of the proof follows as before.

\section{References}

[B] V. S. Borkar, Topics in Controlled Markov Chains, pp. 126-128, Pitman Research Notes in Mathematics, No. 240, Longman Scientific and Technical, Barlow, 1991.

[BG] V.S. Borkar and M. K. Ghosh, Ergodic and adaptive control of nearest-neighbor motions, Math. Control Signals Systems, 4 (1991), 81-98. 